\title{
Genetic causes and management of male infertility
}

\author{
Gavin D. Stormont, Christopher M. Deibert \\ Division of Urologic Surgery, Department of Surgery, University of Nebraska Medical Center, Omaha, NE 68198, USA \\ Contributions: (I) Conception and design: All authors; (II) Administrative support: All authors; (III) Provision of study materials or patients: All \\ authors; (IV) Collection and assembly of data: All authors; (V) Data analysis and interpretation: All authors; (VI) Manuscript writing: All authors; (VII) \\ Final approval of manuscript: All authors. \\ Correspondence to: Christopher M. Deibert, MD. Division of Urologic Surgery, Department of Surgery, University of Nebraska Medical Center, S \\ 42nd St \&, Emile St, Omaha, NE 68198, USA. Email: Christopher.deibert@unmc.edu.
}

\begin{abstract}
Infertility affects approximately $15 \%$ of couples. With infertility such a common problem in a generally healthy age group, complete evaluation is needed of both men and women. Infertility work up for men includes a semen analysis, the results of which suggest various supplemental studies, including karyotype. Karyotype is indicated when a patient has findings on history or physical exam concerning for chromosomal abnormalities, azoospermia, or severe oligospermia (count $<5$ million $/ \mathrm{mL}$ ). The most common chromosomal numerical abnormality found on karyotype is Klinefelter syndrome which is classified as redundant sex chromosomes, with the most common chromosomal arrangement being 47, XXY. If a patient is found to have a chromosomal abnormality such as Klinefelter's, there is still a chance of fertility using testicular sperm extraction and in-vitro fertilization.
\end{abstract}

Keywords: Azoospermia; infertility; karyotype; Klinefelter syndrome; semen analysis.

Submitted Oct 19, 2019. Accepted for publication Mar 11, 2020.

doi: $10.21037 /$ tau.2020.03.34

View this article at: http://dx.doi.org/10.21037/tau.2020.03.34

\section{Introduction}

Infertility is defined as the inability to conceive after at least 12 months of regular unprotected intercourse (1). Unfortunately, difficulty with conception affects about $15 \%$ of couples, with approximately $20 \%$ of these cases being caused by male factor alone with another $30-40 \%$ of infertility due to both a male and female factor (2). With contributions of both male and female partners to infertility, it behooves infertility experts to perform analysis of both partners to determine factors that could be inhibiting fecundity. Male infertility analysis typically begins with a semen analysis, a patient history, and physical examination which will determine next steps in care. This review article focuses on the role of karyotype testing in the male infertility evaluation as karyotype testing in infertility is an important tool to diagnose causes for infertility and can allow proper long-term treatment and counselling to patients.

A karyotype is used to detect chromosomal abnormalities, with the most common finding being normal chromosome complement. The most common abnormalities found in men with azoospermia are chromosomal numerical duplications such as 47,XXY, Klinefelter syndrome, but can also find chromosomal deletions, translocations, inversions, and insertions. Many of these chromosomal abnormalities are associated with infertility and a classification of the chromosomal abnormality using karyotyping can determine how best to counsel and treat patients with infertility.

\section{Body}

\section{History of karyotyping}

Karyotype comes from the Greek karyon for kernel or seed and typos for general form. Chromosomes were first observed in plants in 1842 by Carl Wilhelm von Nägeli (3). The first animal chromosomes were isolated from a salamander by Walther Flemming in 1882 (4). By the 1920 's, the human karyotype had been described as 

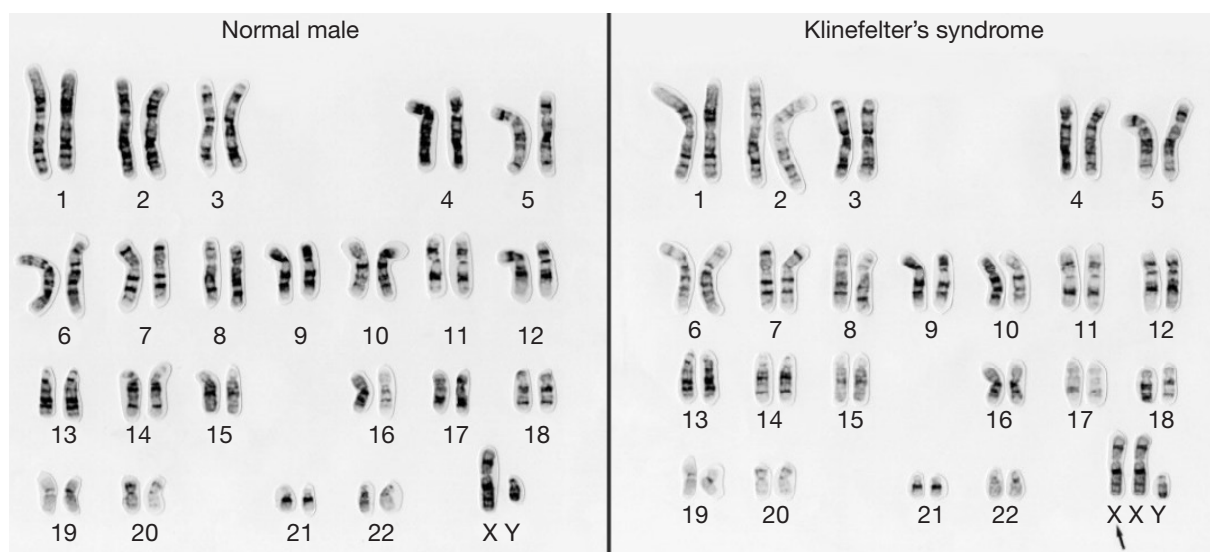

Figure 1 Normal Karyotype. Klinefelter Karyotype. Citation: Wessex Reg. Genetics Centre. CC BY.

48 chromosomes (5). It took another 30 years before Tijo and Levan accurately described the normal diploid human chromosome as 46 (6), this includes 22 pairs of autosomal chromosomes and the 1 pair of sex chromosomes, XY for men (Figure 1). While several types of visual depiction of chromosomes (karyogram) exist, the common visualization of the 46 chromosomes is G-banding. G-banding uses a Giemsa stain which binds DNA causing lighter and darker regions on chromosomes. Darker regions incorporate more stain and are generally adenine and thymine rich regions while cytosine and guanine rich regions are lighter staining. Other types of banding techniques exist but are less often used in karyotyping. These others include as Q-banding which uses a quinacrine stain, R-banding which stains opposite of G-banding, C-banding which uses specialized Giemsa technique that stains heterochromatin (7). For standardization, the human karyotype is presented and numbered in descending order by size from 1 to 22 , with centromeres in the middle and the short $\mathrm{p}$ arm oriented up with the longer q arms down. Knowing this arrangement during the prenatal period can identify at-risk fetuses and guide parental knowledge of chromosomal abnormalities. The next phase of work to develop amniocentesis was done separately by Barr and Gadd from 1956-1967 $(8,9)$. Currently, fetal DNA and sex determination can be detected in circulating maternal blood. Biopsy of IVF created embryos allows detection of aneuploidy and other chromosomal characteristics (10).

\section{Current karyotyping}

A karyotype can be obtained from any cell within the body but is generally performed using blood from a venipuncture. At this time, the cells are cultured and cell division occurs. During culture, the cells are arrested in metaphase using colchicine. Colchicine works by inhibiting the formation of the mitotic spindle preventing dividing cells from progressing to anaphase. This stage of cell division is desirable because the chromosomes are most condensed. These cells are then treated with trypsin causing protein degradation. Following this treatment Giemsa stain is added which selectively binds to chromosomes giving a characteristic pattern to the chromosomes allowing them to be organized into the typical karyogram (Figure 1) from chromosome 1-22 with sex chromosomes labeled at the end. Generally, 15-20 metaphase cells are analyzed into karyograms to ensure standardization and correct preparation of samples (7). This analysis takes about 72 hours for results. Karyotyping generally has a resolution of 5-10 mega bases, making small abnormalities difficult to visualize. Karyotyping is liable to miss mosaicism (when an individual is not made up of a single genotype but is made up of two distinct sets of genotypes) due to the relatively few numbers of karyograms analyzed.

To better characterize mosaicism, fluorescence in situ hybridization (FISH) is used. This study allows more specific analysis of DNA sequences on chromosomes. The technique uses an RNA or DNA probe, single strand of genetic material, with a fluorescent marker. This probe binds to the complementary nucleic acid strain within the DNA molecule. FISH analysis can utilize any cell within the body. The cell mixture denatured to allow the probe access to the DNA. The probe binds to its complimentary DNA and then the fluorescent portion of the probe is activated. FISH is better suited for analysis of mosaicism as it allows for analysis of hundreds of cells instead of 15-20 
as analyzed with karyotyping (11). FISH requires about 24-48 hours for results. It has a resolution of around 3 mega bases and can detect aneuploidy as low as 7\% (12). The increased resolution and ability to detect aneuploidy to as low $7 \%$ unfortunately still poses a risk of false negatives for chromosome abnormalities.

\section{When to karyotype}

During the analysis of infertility in a male, a karyotype is a valuable tool to help determine the cause for infertility. A karyotype is indicated, if there is clinical suspicion of chromosomal abnormality. More defined indications include the classical Klinefelter's presentation: tall stature, gynecomastia, limited facial and body hair, abnormally small testes, azoospermia, and a metabolic evaluation showing increased FSH, LH, and decreased 17-ketosteroids and testosterone (13). The American Society of Reproductive Medicine (ASRM) (14) as well as the Royal College of Obstetricians and Gyanecologist (15) recommend a karyotype for both parents with recurrent pregnancy loss. Recurrent pregnancy loss has been shown to increase structural balanced chromosome abnormalities found from around. Seven percent to $2.2 \%$ and then $4.8 \%$ and $5.2 \%$ after 1, 2, 3 miscarriages respectively (16). Semen analysis is an integral part of infertility analysis especially with respect to performing a karyotype. This is because the rate of chromosomal anomalies in the general male population ranges between $1 / 500$ and 1/1,000 (17) while men with azoospermia or who are severely oligospermic $(<5$ million $/ \mathrm{mL})$ have $13.7 \%$ and $4.6 \%$ rate of chromosomal anomalies respectively (18). The ASRM as well as the American Urologic Association best practice guidelines for Optimal Evaluation of the Infertile Male recommends offering karyotyping and genetic counselling to men with severe oligospermia and azoospermia $(19,20)$. The chromosomal analysis will elucidate chromosomal duplications, deletions, inversions, insertions or translocations, and the couple can be counseled appropriately about the risk to offspring.

Karyotyping can be a costly part of the evaluation and may not be covered by insurance. Cost of karyotype ranges between \$836-\$1,500 (costs from the University of Nebraska). Because of this potential cost, karyotyping should be limited to only individuals that would benefit from this information, based on history, physical, and semen analysis as discussed above.

\section{Various results of karyotypes and chromosomal anomalies}

\section{Normal}

The majority of men with azoospermia and severe oligospermia will have normal karyotypes, $86.3 \%$ and $95.4 \%$ respectively (10). This result may be complicated by rates of mosaicism (an individual that is composed of multiple genotypes instead of one) as this can be missed by karyotyping but which is more readily seen when fluorescence in situ hybridization (FISH) is performed. However, FISH is not utilized frequently. While there may not be a chromosomal abnormality that caused the azoospermia, a genetic cause may still be the culprit, just not one that can be readily tested, and of course not included in a standard karyotype analysis.

\section{Deletions}

Chromosomal deletions can occur on a macroscopic scale as seen with mosaicism or microscopic scale as seen with microscopic $\mathrm{Y}$ chromosomal deletions. 45, X/46, XY mosaicism is seen in $1 / 15,000$ births (21) with $90 \%$ of individuals with $45, \mathrm{X} / 46, \mathrm{XY}$ mosaicism having normal male genitalia (22). Unfortunately, mosaicism will only be identified with usage of FISH analysis or a study of multiple karyograms. The difficulty in this diagnosis is that with mosaicism there is rarely a standardized phenotype to prompt the physician to perform a FISH assay. $45, \mathrm{X} / 46, \mathrm{XY}$ mosaicism presents with a very large spectrum of phenotypes, from individuals that have the characteristic Turner syndrome body habitus to patients with ambiguous genitalia to normal-appearing males with slightly short stature who typically progress through puberty normally (23). Turner mosaicism occurs in 76/100,000 births (24) Because of this normal progression through puberty, these patients may only be diagnosed during an infertility evaluation with a semen analysis showing azoospermia (25). They are found to have normal testosterone levels with elevated gonadotropin levels (25).

Originally macroscopic $\mathrm{Y}$ chromosomal deletions were believed to be the cause of infertility but upon further elucidation specific microscopic $\mathrm{Y}$ chromosomal deletions were noted to be the cause of azoospermia within the region known as azoospermia factor (AZF) (26). AZF deletions are responsible for between $1 \%$ and $18 \%$ of idiopathic male infertility based on geographic location (27). AZF has further been divided into AZF a,b,c (28). Patients with $\mathrm{AZFa}, \mathrm{AZFb}, \mathrm{AZF} \mathrm{b}+\mathrm{c}$ that underwent sperm extraction 
Table 1 Klinefelter karyotypes, prevalence, phenotype, and semen analysis

\begin{tabular}{llll}
\hline Karyotype & Prevalence & Phenotype & Semen analysis \\
\hline $\begin{array}{l}\text { 47,XXY (Orignial Klinefelter 152/100,000 } \\
\text { syndrome) } \\
48, \mathrm{XXYY}\end{array}$ & $\begin{array}{l}\text { Tall, gynecomastia, limited facial and } \\
\text { body hair, small firm testicles }\end{array}$ & Oligospermia, azoospermia \\
$48, \mathrm{XXXY}$ & $1 / 18,000-40,000$ & Similar to $47, \mathrm{XXY}$ & Oligospermia, azoospermia \\
$49, \mathrm{XXXXY}$ & $1 / 50,000$ & Similar to 47,XXY & Oligospermia, azoospermia \\
& $1 / 85,000-100,000$ & $\begin{array}{l}\text { Short, coarse facies, hyperextensible joints, } \\
\text { cryptochidism, intellectual delay, hypotonia }\end{array}$ & Oligospermia, azoospermia \\
$47, \mathrm{XYY}$ & $1 / 1,000$ & Highly variable & Highly variable \\
$48, \mathrm{XYYY}$ & 15 described in literature & Increased height & Typically normal \\
$49, \mathrm{XYYYY}$ & 6 described in literature & Increased height, low set ears, micrognathia & Unknown \\
\hline
\end{tabular}

were not found to have sperm (29) but with AZFc deletion, sperm retrieval is possible, with sperm extraction occurring in $72 \%$ of patients (30). Fortunately, AZFc deletions account for $60 \%$ of total AZF deletions (28).

\section{Duplications}

The most common chromosomal duplication finding for men with non-obstructive azoospermia on karyotype analysis will be 47,XXY (Figure 1). This chromosomal duplication falls into the category of Klinefelter's syndrome which is any karyotype with additional sex chromosomes and has a prevalence of 152 per 100,000 males (31). This has also been found in patients with an apparently normal karyotype, as Klinefelter's can present as mosaics in about $4-12 \%$ of patients with azoospermia and oligospermia (32). Because of this finding, some have advocated for FISH analysis along with karyotype on patients that exhibit azoospermia or oligospermia (32). There are various genotypes of Klinefelter's with the 47,XXY being the most commonly found in approximately $80 \%$. The remaining $20 \%$ are found to have multiple duplications of $\mathrm{X}$ or $\mathrm{Y}$ chromosomes (17).

The most common karyotype variant after Klinefelter's is $47, \mathrm{XYY}$ with a prevalence of about 1/1,000 (33) (Table 1). This presents with variable phenotypes from minimal signs or symptoms to developmental delays and behavioral problems and normal semen analysis to azoospermia. Because of this variable presentation, this syndrome is difficult to clinically suspect (34). 48,XYYY, 15 documented cases (35), is the least clinically concerning karyotype with the main feature being increased height. Typically, normal genitalia were noted, but some individuals are found to have hypogonadism with azoospermia (35). 49,XYYYY, 6 cases documented, exhibit normal genitalia, but height variation, low set ears, and micrognathia (35). 48, XXYY is the third most common karyotype anomaly variant 1:18,000$1: 40,000$ (36). The phenotype is typically similar to Klinefelter's, exhibiting tallness, long thin legs, small testes, sparse body hair, gynecomastia, increased FSH and LH (23). It is noted that with increasing numbers of $\mathrm{X}$ duplications there is a decrease in prevalence of karyotype, 48, XXXY occurring 1:50,000 births while 49,XXXXY occurring between 1:85,000-1:100,000 births (37). 48,XXXY phenotype is also similar to Klinefelter's; tall, decreased upper segment to lower segment ratio, hypertelorism, epicanthic folds, small testes, elevated FSH and LH, gynecomastia (35). 49, XXXXY is more clinically severe and has fewer similarities to Klinefelter's. Patients exhibit coarse facies, short stature, hypotonia, hyperextensible joints, cryptorchidism, elevated FSH and LH, intellectual delay, and synostosis (35). Learning difficulties and behavior abnormalities were noted for all variants, but there was concern that this finding may be due to ascertainment bias, as many of these individuals were karyotyped after hospitalization for behavioral or psychiatric illnesses (35). In individuals with varying duplications of X's, the only common features were hypoplastic testes and elevated FSH and LH.

One can assume that any variety of chromosomal abnormalities exist. While these are likely to be extremely rare there have been case reports. For example, a poly mosaic male was found with 3 different chromosomal genotypes consisting of $47, \mathrm{XYY}(28 \%) / 48, \mathrm{XYYY}$ (68\%)/49,XYYYY (4\%) (38).

\section{Translocations}

Translocations result in re-arrangements of the chromosomal 

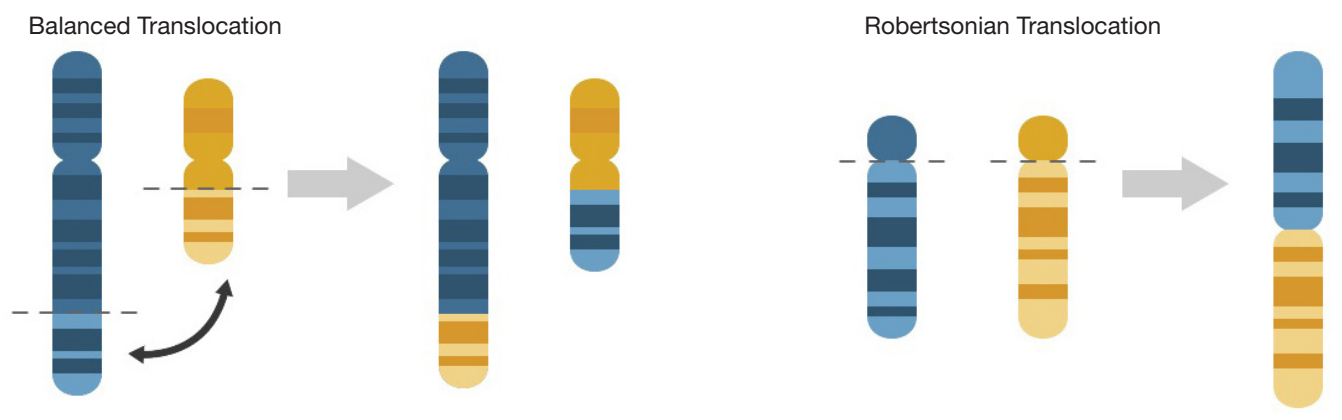

Figure 2 Various translocations of chromosomes. Citation: yourgenome, Genome Research Limited.

material. These can be balanced or unbalanced translocations depending on even or uneven shifting of material (Figure 2). Balanced translocations essentially switch material between chromosomes without any loss or gain of genes and therefore causes no negative imprint or phenotype for the affected individual. These occur in about 1 in 500 births (39). Though typically healthy, these carriers have a higher risk of creating unbalanced translocations in their gametes, which would then be passed on to their child. In an unbalanced translocation, genetic information is exchanged unevenly such that there are either extra or missing genes. A less common exchange is non-reciprocal, in which material from 1 chromosome moves to another, but no material comes back to the first.

Robertsonian translocations occur at or near the centromere of chromosomes 13, 14, 15 and 21, and 22 that have very short arms already. The shorts arms fuse and the long arms form a much larger chromosome (Figure 2). Often the overall chromosome count is reduced from 23 to 22 because of this. It occurs in about 1 in 1,000 live births without phenotypic changes or heighted disease risk (40). However, it does raise the risk of trisomy in any offspring, particularly Down syndrome.

\section{Treatment}

\section{Evaluate partner}

When patients are experiencing problems with infertility both partners should be evaluated. The female infertility work-up generally should be performed prior or concomitantly to the male infertility evaluation. The female work-up typically is conducted by an obstetrician/ gynecologist with experience in reproductive medicine.

\section{Optimize hormones}

When a male is found to have hormonal abnormalities during an infertility work-up, optimization is required to ensure the greatest chances of fertility. The site of deficiency determines which type of treatment is needed. If normal FSH/LH and testosterone, hormone treatment would be of no benefit. If FSH/LH is normal and testosterone is low or hypergonadotropic hypogonadism, which is typically found with Klinefelter syndrome, there is minimal benefit in treatment as increasing FSH/LH would not stimulate atrophic testicles. Exogenous testosterone does not confer benefit for spermatozoa production (41). If hypogonadotropic hypogonadism is found, then clomiphene citrate or HCG can be used to correct the abnormality (42). If a testosterone/estradiol ratio is $<10$, then anastrozole can be used to increase this ratio by blocking conversion of testosterone to estradiol (43).

\section{Sperm extraction}

Because non-obstructive azoospermia is seen on semen analysis of Klinefelter's patients, the main method to achieve fertility is to utilize sperm extraction and intracytoplasmic sperm injection. There are two types of testicular sperm extraction techniques: microscopic and non-microscopic with the sperm extraction rates of $57 \%$ and $42 \%$ respectively (43). Pregnancy rates were $36 \%$ for both types of extraction (44). Extraction has been found to be most successful in the age group 16-30 when compared to individuals younger than 16 (44). Prior to the procedure, it is a generally accepted practice to discontinue testosterone replacement. Many hypothesize and recommend hormonal optimization, but unfortunately there are no studies that compare hormonal treatment with controls.

\section{Conclusions}

Chromosomal abnormalities are relatively common in men with infertility. Therefore, men with azoospermia, 
severe oligospermia, or clinical phenotypes suggestive of chromosomal abnormalities should have a karyotype performed. Treatment varies pending the results of the chromosomal analysis.

\section{Future directions}

While current urologic intervention can provide a pathway to fertility for some patients with chromosomal abnormalities, research and development continues to provide promising possibilities for these individuals. For example, Clustered regularly interspaced short palindromic repeats (CRISPR) technology is a gene editing tool. CRISPR allows removal of genes in chromosomes but also has been used to eliminate complete autosomal and sex chromosomes (45). This is important because it may allow individuals with virtually any chromosomal abnormalities to have the chromosomal abnormality repaired, hopefully improving chances of fertility as well as other aspects of their daily life made challenging by their genetic abnormality (46).

\section{Acknowledgments}

Funding: None.

\section{Footnote}

Provenance and Peer Review: This article was commissioned by the Guest Editors (Keith Jarvi and Jared Bieniek) for the series "Genetic Causes and Management of Male Infertility" published in Translational Andrology and Urology. The article has undergone external peer review.

Conflicts of Interest: Both authors have completed the ICMJE uniform disclosure form (available at http://dx.doi. org/10.21037/tau.2020.03.34). The series "Genetic Causes and Management of Male Infertility" was commissioned by the editorial office without any funding or sponsorship. The authors have no other conflicts of interest to declare.

Etbical Statement: The authors are accountable for all aspects of the above work in ensuring that questions related to the accuracy or integrity of any part of the work are appropriately investigated and resolved.

Open Access Statement: This is an Open Access article distributed in accordance with the Creative Commons
Attribution-NonCommercial-NoDerivs 4.0 International License (CC BY-NC-ND 4.0), which permits the noncommercial replication and distribution of the article with the strict proviso that no changes or edits are made and the original work is properly cited (including links to both the formal publication through the relevant DOI and the license). See: https://creativecommons.org/licenses/by-nc-nd/4.0/.

\section{References}

1. Zegers-Hochschild F, Adamson GD, Dyer S, et al. The International Glossary on Infertility and Fertility Care, 2017. Hum Reprod 2017;32:1786-801.

2. Thonneau P, Marchand S, Tallec A, et al. Incidence and main causes of infertility in a resident population (1,850,000) of three French regions (1988-1989). Hum Reprod 1991;6:811-6.

3. Bahadur B, Rajam MV, Sahijram L, et al. Plant Biology and Biotechnology Volume II: Plant Ge-nomics and Biotechnology. New Delhi: Springer India; 2015.

4. Walther Flemming. Kiel University I Famous Scholars from Kiel. Available online: https://www.uni-kiel.de/ grosse-forscher/index.php?nid=flemming\&lang=e. Accessed December 14, 2019.

5. Painter TS. Studies in mammalian spermatogenesis. II. The spermatogenesis of man. Journ Exper Zool 1923;37:291-321.

6. Tjio JH, Levan A. The Chromosome Number of Man. Problems of Birth Defects 1956:112-8.

7. Nature News. Available online: https://www.nature. com/scitable/topicpage/karyotyping-for-chromosomalabnormalities-298/. Accessed December 14, 2019.

8. Barr ML. Prenatal Sex Determination. Can Med Assoc J 1956;74:922-3.

9. Philipp EE, Barnes J, Newton M. Scientific Foundations of Obstetrics and Gynaecology. William Heinemann Medical Books 1970; 285-91.

10. Ethics Committee of the American Society for Reproductive Medicine. Electronic address: asrm@ asrm.org; Ethics Committee of the American Society for Reproductive Medicine. Disclosure of sex when incidentally revealed as part of preimplantation genetic testing (PGT): An Ethics Committee Opinion. Fertil Steril 2018;110:625-7.

11. Nature News. Available online: https://www.nature.com/ scitable/topicpage/fluorescence-in-situ-hybridizationfish-327/. Accessed December 14, 2019.

12. Vermeesch JR, Fiegler H, Leeuw ND, et al. Guidelines for 
molecular karyotyping in constitu-tional genetic diagnosis. Eur J Hum Genet 2007;15:1105-14.

13. Klinefelter HF, Reifenstein EC, Albright F. Syndrome Characterized by Gynecomastia, Asper-matogenesis without A-Leydigism, and Increased Excretion of FollicleStimulating Hormone1. J Clin Endocrinol Metab 1942;2:615-27.

14. Practice Committee of the American Society for Reproductive Medicine. Evaluation and treatment of recurrent pregnancy loss: a committee opinion. Fertil Steril 2012;98:1103-11.

15. The investigation and treatment of couples with recurrent first-trimester and second-trimester miscarriage Clinical Practice Guidelines. Guideline Central. Available online: https://www.guidelinecentral.com/summaries/theinvestigation-and-treatment-of-couples-with-recurrentfirst-trimester-and-second-trimester-miscarriage/\#sectionsociety. Accessed December 14, 2019.

16. Daniel A, Hook EB, Wulf G. Risks of unbalanced progeny at amniocentesis to carriers of chromosome rearrangements: Data from United States and Canadian laboratories. Am J Med Genet 1989;33:14-53.

17. Forti G, Corona G, Vignozzi L, et al. Klinefelter's syndrome: a clinical and therapeutical update. Sex Dev 2010;4:249-58.

18. Van Assche E, Bonduelle $M$, Tournaye $\mathrm{H}$, et al. Cytogenetics of infertile men. Hum Reprod 1996;11 Suppl 4:1-24; discussion 25-6.

19. Optimal Evaluation of the Infertile Male. Optimal Evaluation of the Infertile Male - American Urological Association. https://www.auanet.org/guidelines/maleinfertility-optimal-evaluation-best-practice-statement. Accessed December 14, 2019.

20. Practice Committee of the American Society for Reproductive Medicine. Diagnostic evaluation of the infertile male: a committee opinion. Fertil Steril 2015;103:e18-25.

21. Nielsen J, Wohlert M. Chromosome abnormalities found among 34,910 newborn children: results from a 13year incidence study in Arhus, Denmark. Hum Genet 1991;87:81-3.

22. Hsu LYF. Phenotype/Karyotype correlations of $Y$ chromosome aneuploidy with emphasis on structural aberrations in postnatally diagnosed cases. Am J Med Genet 1994;53:108-40.

23. Johansen ML, Hagen CP, De Meyts ER, et al. 45,X/46,XY Mosaicism: Phenotypic Characteris-tics, Growth, and Reproductive Function-A Retrospective Longitudinal
Study. J Clin Endocrinol Metab 2012;97:1540-9.

24. Tuke MA, Ruth KS, Wood AR, et al. Mosaic Turner syndrome shows reduced phenotypic pene-trance in an adult population study compared to clinically ascertained cases. Genet Med 2017. doi: 10.1101/177659.

25. Akinsal EC, Baydilli N, Bayramov R, et al. A Rare Cause of Male Infertility: 45,X/46,XY Mosaicism. Urol Int 2018;101:481-5.

26. Hartung M, Devictor M, Codaccioni JL, et al. Yq deletion and failure of spermatogenesis. Ann Genet 1988;31:21-6.

27. Stahl PJ, Masson P, Mielnik A, et al. A decade of experience emphasizes that testing for $\mathrm{Y}$ mi-crodeletions is essential in American men with azoospermia and severe oligozoospermia. Fertility and Sterility 2010;94:1753-6.

28. Bardoni B, Zuffardi O, Guioli S, et al. A deletion map of the human Yq11 region: implications for the evolution of the $\mathrm{Y}$ chromosome and tentative mapping of a locus involved in spermato-genesis. Genomics 1991;11:443-51.

29. Hopps CV. Detection of sperm in men with $Y$ chromosome microdeletions of the AZFa, AZFb and AZFc regions. Hum Reprod 2003;18:1660-5.

30. Ferlin A, Arredi B, Speltra E, et al. Molecular and Clinical Characterization of $\mathrm{Y}$ Chromosome Microdeletions in Infertile Men: A 10-Year Experience in Italy. J Clin Endocrinol Metab 2007;92:762-70.

31. Bojesen A, Juul S, Gravholt CH. Prenatal and Postnatal Prevalence of Klinefelter Syndrome: A National Registry Study. J Clin Endocrinol Metab 2003;88:622-6.

32. Elghezal H, Hidar S, Braham R, et al. Chromosome abnormalities in one thousand infertile males with nonobstructive sperm disorders. Fertil Steril 2006;86:1792-5.

33. Robinson DO, Jacobs PA. The Origin of the Extra $Y$ Chromosome in Males with a 47,XYY Kary-otype. Hum Mol Genet 1999;8:2205-9.

34. Kim IW, Khadilkar AC, Ko EY, et al. 47,XYY Syndrome and Male Infertility. Rev Urol 2013;15:188-96.

35. Abedi M, Salmaninejad A, Sakhinia E. Rare 48, XYYY syndrome: case report and review of the literature. Clin Case Rep 2017;6:179-84.

36. Sørensen K, Nielsen J, Jacobsen P, et al. The 48,XXYY syndrome. J Ment Defic Res 1978;22:197-205.

37. Kleczkowska A, Fryns JP, Van den Berghe H. $\mathrm{X}$-chromosome polysomy in the male. The Leu-ven experience 1966-1987. Hum Genet 1988;80:16-22.

38. Gigliani F, Gabellini P, Marcucci L, et al. Peculiar mosaicism 47,XYY/48,XYYY/49,XYYYY in man. J Genet Hum 1980;28:47-51. 
39. Mackie Ogilvie C, Scriven PN. Meiotic outcomes in reciprocal translocation carriers ascertained in 3-day human embryos. Eur J Hum Genet 2002;10:801-6.

40. Therman E, Susman B, Denniston C. The nonrandom participation of human acrocentric chromosomes in Robertsonian translocations. Annals of Human Genetics 1989;53:49-65

41. Khourdaji I, Lee H, Smith RP. Frontiers in hormone therapy for male infertility. Transl Androl Urol 2018;7:S353-66.

42. Dabaja AA, Schlegel PN. Medical treatment of male infertility. Transl Androl Urol 2014;3:9-16.

43. Aksglaede L, Juul A. Therapy of endocrine disease:

Cite this article as: Stormont GD, Deibert CM. Genetic causes and management of male infertility. Transl Androl Urol 2021;10(3):1365-1372. doi: 10.21037/tau.2020.03.34
Testicular function and fertility in men with Klinefelter syndrome: a review. Eur J Endocrinol 2013;168:R67-76.

44. Franik S, Hoeijmakers Y, Dhauwers K, et al. Klinefelter syndrome and fertility: sperm preser-vation should not be offered to children with Klinefelter syndrome. Hum Reprod 2016;31:1952-9.

45. Zuo E, Huo X, Yao X, et al. CRISPR/Cas9-mediated targeted chromosome elimination. Genome Biol 2017;18:224.

46. Pandey VK, Tripathi A, Bhushan R, et al. Application of CRISPR/Cas9 Genome Editing in Genetic Disorders: A Systematic Review Up to Date. J Genet Syndr Gene Ther 2017;8:321. 